\title{
Theory of Fano resonances in graphene: The influence of orbital and structural symmetries on STM spectra
}

\author{
T. O. Wehling, ${ }^{1}$ H. P. Dahal, ${ }^{2}$ A. I. Lichtenstein, ${ }^{1}$ M. I. Katsnelson, ${ }^{3}$ H. C. Manoharan, ${ }^{4,5}$ and A. V. Balatsky ${ }^{2,6}$ \\ ${ }^{1}$ I. Institut für Theoretische Physik, Universität Hamburg, D-20355 Hamburg, Germany \\ ${ }^{2}$ Theoretical Division, Los Alamos National Laboratory, Los Alamos, New Mexico 87545, USA \\ ${ }^{3}$ Institute for Molecules and Materials, Radboud University Nijmegen, NL-6525 AJ Nijmegen, The Netherlands \\ ${ }^{4}$ Department of Physics, Stanford University, Stanford, California 94305, USA \\ ${ }^{5}$ Stanford Institute for Materials and Energy Science, Menlo Park, California 94025, USA \\ ${ }^{6}$ Center for Integrated Nanotechnologies, Los Alamos National Laboratory, Los Alamos, New Mexico 87545, USA
}

(Received 25 November 2009; published 9 February 2010)

\begin{abstract}
We theoretically investigate Fano factors arising in local spectroscopy of impurity resonances in graphene. It is demonstrated that Fano line shapes can strongly differ from the antiresonances usually found on metal surfaces. Graphene's highly symmetric Fermi points make this effect particularly sensitive to the detailed atomistic structure and orbital symmetries of the impurity. After a model discussion based on an Anderson impurity coupled to an electron bath with linearly vanishing density of states, we present first-principles calculations of Co adatoms on graphene. For Co above the center of a graphene hexagon, we find that the two-dimensional $E_{1}$ representation made of $d_{x z}, d_{y z}$ orbitals is likely responsible for the hybridization and ultimately Kondo screening for cobalt on graphene. Anomalously large Fano $q$ factors depending strongly on the orbitals involved are obtained. For a resonant $s$-wave impurity, a similarly strong adsorption site dependence of the $q$ factor is demonstrated. These anomalies are striking examples of quantum-mechanical interference related to the Berry phase inherent to the graphene band structure.
\end{abstract}

DOI: 10.1103/PhysRevB.81.085413

PACS number(s): 73.20.Hb, 72.10.Fk, 73.20.At

\section{INTRODUCTION}

Scanning tunneling microscopy (STM) allows us to probe the electronic properties of conducting materials with atomic scale spatial resolution. This experimental technique is particularly well suited to study electronic correlation phenomena. One of the most famous correlation phenomena is the Kondo effect arising from a localized magnetic moment being screened by the conduction electrons. ${ }^{1}$ It results in a formation of a sharp Abrikosov-Suhl resonance in local density of states (LDOS) at the Fermi level below a characteristic Kondo temperature $T_{\mathrm{K}}$. Today, the Kondo effect is well understood for impurities in bulk materials and simple model systems. However, large interest in this phenomenon has been revived recently since STM has been revealing the intricacies of Kondo effect at metal surfaces, caused by $d$ and $f$ adatoms. ${ }^{2-4}$ The rich electronic structure of threedimensional metals like $\mathrm{Cu}$, in general, controls impurity effects at surfaces ${ }^{5-7}$ and the effects turned out to depend strongly on atomistic details. This requires a realistic description of the magnetic impurity, e.g., taking into account the detailed bonding geometry, to understand the electronic spectra of these structures.

Graphene-a monolayer of carbon atoms arranged in a honeycomb lattice-is the first truly two-dimensional material ${ }^{8}$ and provides a two-dimensional electron gas with a distinct and highly symmetric low-energy electronic structure: at two nonequivalent corners of the Brillouin zone, $K$ and $K^{\prime}$, the linearly dispersing valence and conduction bands touch forming a conical point with nontrivial Berry phase $\pi \cdot{ }^{9,10}$ Thus, electronic excitations in graphene resemble massless Dirac fermions with the speed of light being replaced with the Fermi velocity $v_{\mathrm{f}} \approx c / 300$. Therefore, graphene provides a model system for understanding quantum effects in reduced dimensions and in the presence of an "ultrarelativistic" conduction-electron bath.

Theoretical studies have shown that even in undoped graphene the Kondo effect can exist above a certain critical coupling despite the linearly vanishing density of states, ${ }^{11-15}$ a situation very similar to magnetic impurities in the pseudogap phase of high- $T_{c}$ superconductors. ${ }^{11,12,16}$ Moreover, backgating ${ }^{8}$ as well as chemical doping ${ }^{17,18}$ allows one to control the chemical potential in graphene and to tune Kondo physics and electron tunneling in this way.

In this paper, we address how impurity resonances, in general, and Kondo resonances, in particular, manifest in STM experiments on graphene. It is shown why Fano resonances in the STM spectra can depend unusually strongly on the chemical potential, on the involved impurity orbitals, as well as the real-space position of the impurity. To this end, we first consider the single impurity Anderson model with graphene providing the host electronic structure and compare to a simple model of a usual metal surface. With this background, we turn to a more realistic ab initio based description of magnetic impurities on graphene and discuss the case of Co adatoms as in the recent experiment by Manoharan et al. ${ }^{19}$ By comparison to $\mathrm{Co}$ on $\mathrm{Cu}$ (111), an extensively studied system, ${ }^{5-7}$ possessing also hexagonal symmetry of the surface, we will demonstrate the importance of impurityinduced resonances in graphene ${ }^{20}$ and the particular sensitivity of the $q$ factor to orbital symmetries. Furthermore, we analyze model $s$-wave impurities being bound to different sites of the graphene lattice and show that a strong adsorption site dependence of Fano factors in STM experiments can be expected there. 


\section{MODEL FOR TUNNELING INTO IMPURITY RESONANCES}

The $\pi$-band the tight-binding Hamiltonian of graphene reads as ${ }^{21}$

$$
\hat{H}_{0}=-t \sum_{\langle i, j\rangle}\left(a_{i}^{\dagger} b_{j}+b_{j}^{\dagger} a_{i}\right)
$$

where $a_{i}$ and $b_{i}$ are the Fermi operators of electrons in the carbon $p_{z}$ orbital of sublattice atoms $\mathrm{A}$ and $\mathrm{B}$ in the cell at $R_{i}$, respectively. The sum includes all pairs of nearest-neighbor carbon atoms and $t \approx 2.7 \mathrm{eV}$ is the hopping parameter. With the Fourier-transformed operators $a_{k}\left(b_{k}\right)$, defined by $a_{i}$ $=\int_{\Omega_{B}}\left(d^{2} k / \Omega_{B}\right) e^{i k R_{i}} a_{k}$ and $b_{i}$ analogously, the Hamiltonian can be rewritten as

$$
\hat{H}_{0}=\int_{\Omega_{B}} \frac{d^{2} k}{\Omega_{B}} \Psi_{k}^{\dagger} H_{k} \Psi_{k}, \quad \text { with } \Psi(k)=\left[\begin{array}{l}
a_{k} \\
b_{k}
\end{array}\right] .
$$

$H_{k}$ is the $k$-dependent $2 \times 2$ matrix

$$
H_{k}=\left(\begin{array}{cc}
0 & \xi(k) \\
\xi^{*}(k) & 0
\end{array}\right),
$$

where $\xi(k)=-t \sum_{j=1}^{3} e^{i k\left(b_{j}-b_{1}\right)}$, with $b_{j}(j=1,2,3)$ being the vectors connecting neighboring atoms ${ }^{22}$ and $\Omega_{B}$ being the area of the Brillouin zone. An impurity contributing a localized orbital, $\hat{H}_{\text {imp }}=\epsilon_{\text {imp }} \Sigma_{\sigma} d_{\sigma}^{\dagger} d_{\sigma}+U n_{\uparrow} n_{\downarrow}$, with Fermi operator $d$, energy $\epsilon_{\text {imp }}$, and on-site Coulomb repulsion $U$, is considered. Its hybridization with the graphene bands is described by $\hat{V}=\sum_{k, \sigma} \Psi_{k, \sigma}^{\dagger} V_{k d} d_{\sigma}+$ H.c. This problem has been extensively discussed for normal metals and is usually called the "Anderson impurity model."1

In this framework, the connection between a tip and a sample in the STM experiment can be expressed by the transfer Hamiltonian

$$
M=\sum_{\sigma}\left(M_{d t} d_{\sigma}^{\dagger} t_{\sigma}+\text { H.c. }\right)+\sum_{k \sigma}\left(\Psi_{k \sigma}^{\dagger} M_{k t} t_{\sigma}+\text { H.c. }\right)
$$

describing tunneling of electrons from and to the STM tip with the tunneling matrix elements $M_{d t}$ and $M_{k t}$ and the Fermi operators $t_{\sigma}\left(t_{\sigma}^{\dagger}\right)$ for electrons in the STM tip.

The Fano $q$ factor in the STM $d I / d V$ spectra can be understood in terms of this model by using the equation of motion approach from Ref. 23. One finds

$$
q=\frac{A}{B}
$$

with

$$
\begin{gathered}
A=M_{d t}+\sum_{k} V_{d k} M_{k t} P\left(\frac{1}{E_{\mathrm{F}}-\epsilon_{k}}\right), \\
B=-\pi \sum_{k} V_{d k} M_{k t} \delta\left(E_{\mathrm{F}}-\epsilon_{k}\right),
\end{gathered}
$$

where $P$ is the Cauchy principle value symbol.

To obtain qualitative insights we proceed by simplifying these expressions in close analogy to Eqs. (2.7)-(2.10) of Ref. 24: for each conduction-electron wave function $\Psi_{k}(r)$, we rewrite the hopping matrix elements to the impurity and to the tip according to

$$
M_{k t} \approx M_{0} \Psi_{k}^{*}\left(R_{t}\right), \quad V_{d k} \approx V_{0} \Psi_{k}^{*}\left(R_{d}\right),
$$

where $R_{t}$ and $R_{d}$ are the positions of the tip and the impurity, respectively. The matrix elements $M_{0}\left(V_{0}\right)$ contain the dependence on the symmetry of the atomic orbitals of the tip (impurity). We further assume that the wave functions can be factorized as $\Psi_{k}(r)=\phi_{k}\left(r_{\|}\right) f(z)$, where $r=\left(r_{\|}, z\right)$ is split up into a lateral part $r_{\|}$and a vertical coordinate $z$. We consider the case of the tip being directly above the Co impurity, i.e., $R_{t}=\left(r_{\|}, z_{t}\right)$ and $R_{d}=\left(r_{\|}, z_{d}\right)$, which leads to

$$
V_{d k} M_{k t}=V_{0}^{*} M_{0} \Psi_{k}^{*}\left(R_{t}\right) \Psi_{k}\left(R_{d}\right)=\left|\Psi_{k}\left(R_{d}\right)\right|^{2} M V
$$

with $M V=V_{0}^{*} M_{0} f^{*}\left(z_{t}\right) / f^{*}\left(z_{d}\right)$ independent of $k$. Thus, Eqs. (6) and (7) yield

$$
A=M_{d t}+M V \operatorname{Re} G\left(E_{\mathrm{F}}\right), \quad B=M V \operatorname{Im} G\left(E_{\mathrm{F}}\right)
$$

with the local conduction-electron Green's function $G(E)$ $=\Sigma_{k}\left[\left|\Psi_{k}\left(R_{d}\right)\right|^{2} /\left(E-\epsilon_{k}-i 0^{+}\right)\right]$at the impurity site. As argued in Ref. 7, this simple model has proved successful to describe Fano factors for $\mathrm{CoCu}_{n}$ clusters on $\mathrm{Cu}$ (111) and will be used here to understand Fano resonances in graphene and to show why they are different to metals like $\mathrm{Cu}$.

\section{ENERGY AND ORBITAL DEPENDENCE OF THE ASYMMETRY FACTOR}

Usually, the magnetic orbitals of the adatom are strongly localized resulting in $\left|M_{d t}\right| \ll\left|M_{k t}\right|$ and, consequently,

$$
q \approx-\operatorname{Re} G\left(E_{\mathrm{F}}\right) / \operatorname{Im} G\left(E_{\mathrm{F}}\right) .
$$

In a metal with bandwidth $D$ and constant DOS in the vicinity of the impurity, $\operatorname{Im} G(\epsilon)=-\pi / 2 D$ if $-D<\epsilon<D$, we obtain $q \approx \frac{1}{\pi} \ln \left|\left(D+E_{\mathrm{F}}\right) /\left(D-E_{\mathrm{F}}\right)\right| \approx 2 E_{\mathrm{F}} / \pi D$. Hence, $|q|<1$ and the Kondo effect manifests in STM on normal metals as antiresonance close to $E_{\mathrm{F}}$ as long as $\left|M_{d t}\right| \ll\left|M_{k t}\right|$. This is very different for graphene.

The graphene DOS is $N_{0}^{\mathrm{g}}(E)=\left(|E| / D^{2}\right) \Theta(D-|E|)$ resulting in $G(E)=\left(E / D^{2}\right) \ln \left|E^{2} /\left(D^{2}-E^{2}\right)\right|-i \pi N_{0}^{\mathrm{g}}(E)$ and

$$
q \approx \frac{2 \operatorname{sign}\left(E_{\mathrm{F}}\right)}{\pi} \ln \left|\frac{E_{\mathrm{F}}}{D}\right| .
$$

This result follows directly from the linearity of $N_{0}^{\mathrm{g}}(E)$ and the Kramers-Kronig relations. As $D \approx 6 \mathrm{eV}$ (Ref. 25) and usually $E_{\mathrm{F}} \lesssim 0.5 \mathrm{eV}$, the $q$ factor can be $q \gg 1$ and the Kondo effect may manifest in STM as resonance instead of an antiresonance, even for $\left|M_{d t}\right| \ll\left|M_{k t}\right|$. This is in contrast to a normal metal, where predominant tunneling into the conduction-electron states results in a Kondo antiresonance in STM. Moreover, Eq. (11) demonstrates that the $q$ factor in graphene can be expected to depend strongly on the chemical potential.

Any impurity being coupled to graphene leads to characteristic resonances in the local density of states in the vicinity of the impurity. ${ }^{20}$ Moreover, as the real part of the Green's function enters Eq. (10), the $q$ factor carries information about high-energy features of the local electronic structure, 

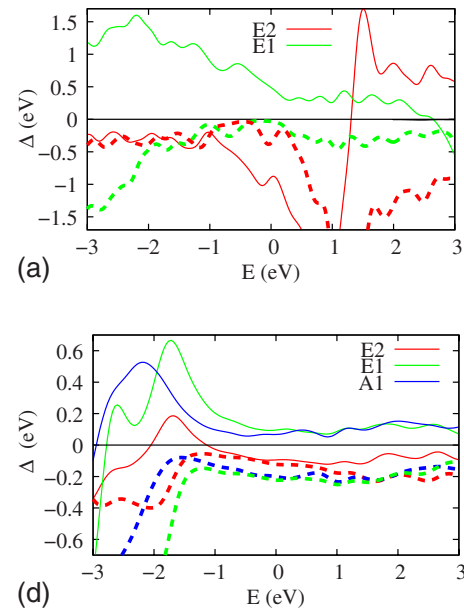
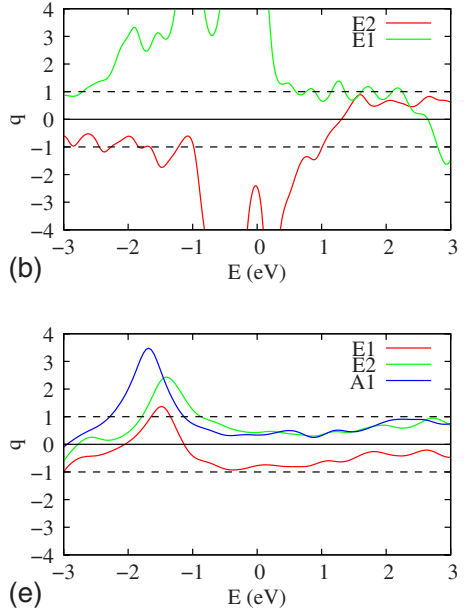

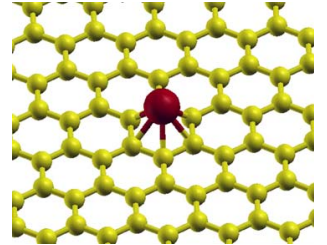

(c)

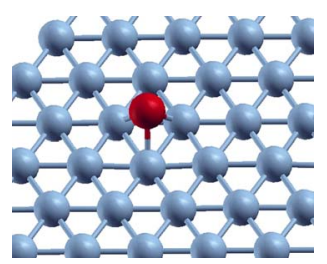

(f)

FIG. 1. (Color online) (a) and (d) Hybridization functions $\Delta$, and (b) and (e) asymmetry factors $q$ for Co on graphene (upper panel) and $\mathrm{Co}$ on $\mathrm{Cu}$ (111) (lower panel) as obtained from our first-principles calculations. The $q$ factors have been calculated from the hybridization functions using Eq. (15). For the hybridization functions $\operatorname{Re} \Delta$ is plotted as solid line, and $\operatorname{Im} \Delta$ as dashed line. The energy $E=0$ corresponds to the Fermi level of the undoped system. The hybridization of Co on graphene is strongly energy and orbital dependent. The adsorption geometries of $\mathrm{Co}$ on graphene and $\mathrm{Cu}(111)$ are shown in (c) and (f), respectively. For the $\mathrm{Cu}$ (111) slabs, only the uppermost $\mathrm{Cu}$ layer is shown for clarity.

which is in general beyond effective low-energy theories. To understand the role of such contributions to the $q$ factor, we illustrate the situation of a realistic impurity by comparing the experimentally important cases of Co on graphene and $\mathrm{Co}$ on $\mathrm{Cu}$ (111).

For a realistic description of these systems we performed density-functional calculations within the generalized gradient approximation (GGA) (Ref. 26) on $6 \times 6$ graphene supercells containing one $\mathrm{Co}$ adatom as well as on $\mathrm{Cu}$ (111) slabs containing five $\mathrm{Cu}$ layers and one $\mathrm{Co}$ adatom. The Vienna $a b$ initio simulation package (VASP) (Ref. 27) with the projector augmented wave (PAW) (Refs. 28 and 29) basis sets has been used for solving the resulting Kohn-Sham equations. In this way we obtained relaxed structures for both systems. In particular, we found that GGA predicts Co to sit above the middle of a hexagon on graphene, with Co on top of carbon and Co above a bridge site being 0.6 and $0.5 \mathrm{eV}$, respectively, higher in energy. This prediction of Co above the middle of a hexagon is in line with previous DFT calculations. ${ }^{30,31}$ The following discussion will be based on the fully relaxed structure minimum-energy structure of Co above the middle of a hexagon as shown in Fig. 1.

To estimate the Fano $q$ factors we extracted the orbitalresolved Green's functions at the impurity site using atomic orbitals naturally included in the PAW basis sets: the projectors $\left\langle d_{i} \mid \psi_{n \mathbf{k}}\right\rangle$ of orbitals $\left|d_{i}\right\rangle$ localized at the impurity atoms onto the Bloch eigenstates of the Kohn-Sham problem $\left|\psi_{n \mathbf{k}}\right\rangle$ are available when using PAW as implemented in the VASP and these give the local Green's functions according to

$$
G_{i j}(\epsilon)=\sum_{n \mathbf{k}} \frac{\left\langle d_{i} \mid \psi_{n \mathbf{k}}\right\rangle\left\langle\psi_{n \mathbf{k}} \mid d_{j}\right\rangle}{\epsilon+i \delta-\varepsilon_{n \mathbf{k}}} .
$$

So, we employ here the same representation of localized orbitals as used within the local-density approximation (LDA) plus Hubbard $U(\mathrm{LDA}+\mathrm{U})$ scheme implemented in the VASP code itself or as discussed in the context of LDA+dynamical mean field theory (LDA+DMFT) in Ref. 32.

The local Green's functions at the impurity sites as defined in Eq. (12) are $5 \times 5$ matrices, which can used to obtain the hybridization function $\Delta(\epsilon)$ of the impurity,

$$
G^{-1}(\epsilon)=\epsilon+i \delta-\epsilon_{d}-\Delta(\epsilon),
$$

where $\epsilon_{d}$ is the static crystal field and a $5 \times 5$ matrix, in general. Hence, $\Delta(\epsilon)$ are also $5 \times 5$ matrices describing the hybridization of five $d$ electrons of Co. In the particular case of Co above a graphene hexagon, the $C_{6 v}$ symmetry ensures that these matrices are diagonal and decompose into degenerate blocks transforming under $C_{6 v}$ according to the twodimensional $E_{1}$ and $E_{2}$ representations as well as the onedimensional $A_{1}$ representation. The $E_{1}$ and $E_{2}$ components of the hybridization function are depicted in Fig. 1(a). At energies close to the Fermi level of graphene all graphene states are in the vicinity of the two Dirac points. These states transform under $C_{6 v}$ according to $E_{1}$ and $E_{2}$. Hence, the hybridization of the $A_{1}$ impurity orbital to the graphene bands is strongly suppressed. Moreover, the crystal-field splitting appears to be such that the $E_{1}$ orbitals $\left(d_{x z}\right.$ and $\left.d_{y z}\right)$ are highest in energy by approximately $0.5-1 \mathrm{eV}$ as compared to the other $d$ orbitals. So, the $E_{1}$ orbitals are expected to determine the $q$ factor in STM experiments probing the Kondo effect of Co on graphene. $\mathrm{Cu}(111)$ is also a hexagonal surface and the adsorption geometry of $\mathrm{Co}$ on $\mathrm{Cu}(111)$ is $C_{3 v}$ symmetric. The decomposition of the hybridization function of $\mathrm{Co}$ on $\mathrm{Cu}$ (111) into $E_{1}, E_{2}$, and $A_{1}$ orbitals is shown in Fig. 1(c). In contrast to graphene, these hybridization functions are only weakly energy dependent in the vicinity of the Fermi level.

Moreover, for $\mathrm{Co}$ on graphene we have $|\operatorname{Im} \Delta(\epsilon)|$ $\ll|\operatorname{Re} \Delta(\epsilon)|$ in the vicinity of the Fermi level of the undoped system, $\epsilon=0$. This is very different from the case of $\mathrm{Cu}$, where $|\operatorname{Im} \Delta(\epsilon)|$ and $|\operatorname{Re} \Delta(\epsilon)|$ are mainly on the same order. Using 


$$
\Delta(\epsilon)=\sum_{k} \frac{\left|V_{d k}\right|^{2}}{\epsilon+i \delta-\epsilon_{k}} \approx\left|V_{0}\right|^{2} \sum_{k} \frac{\left|\Psi_{k}\left(R_{d}\right)\right|^{2}}{\epsilon+i \delta-\epsilon_{k}},
$$

in combination with Eqs. (8) and (9) and $\left|M_{d t}\right| \ll|M|$, we arrive at

$$
q\left(E_{\mathrm{F}}\right) \approx \operatorname{Re} \Delta\left(E_{\mathrm{F}}\right) / \operatorname{Im} \Delta\left(E_{\mathrm{F}}\right) .
$$

Within this approximation the projectors and eigenenergies obtained from DFT allow for an $a b$ initio prediction of $q$ factors. Figures 1(b) and 1(d) show the $q$ factors predicted for channels of different $C_{6 v} / C_{3 v}$ symmetries as calculated for $\mathrm{Co}$ on graphene and $\mathrm{Cu}$, respectively, as functions of the resonant energy $E$. The calculated $q$ factors for $\mathrm{Co}$ on $\mathrm{Cu}$ (111) are typically on the order of $q \lessgtr 1$ without pronounced energy dependence. This is in contrast to graphene, where $q>1$ in a wide energy range and $q$ is strongly energy dependent. So, $q$ is expected to be strongly sensitive to local changes in the chemical potential of graphene, which can be caused by gate voltages, chemical doping, or substrate effects. Apart from $|q| \gg 1$ for $E \rightarrow 0$, we find the $q$ factor for resonances with $E_{1}$ and $E_{2}$ symmetries displaying markedly different and asymmetric energy dependences, which are effects well beyond a linearized dispersion analysis.

\section{SITE DEPENDENCE OF HYBRIDIZATION MATRIX ELEMENTS}

For Co on graphene, we saw that graphene's Fermi surface being made up of states transforming as $E_{1}$ and $E_{2}$ under $C_{6 v}$ leads to particular Co orbitals being decoupled from the graphene bands. This special symmetry of graphene's Fermi surface makes the $q$ factors seen in Kondo resonances in scanning tunneling spectroscopy particularly dependent of the precise atomic arrangement of the magnetic impurity. Similar to the sensitivity of the Fano resonances to orbital symmetries, the $q$ factor can depend strongly on the adsorption site of the impurity. This can be illustrated by the site dependence of the $q$ factors for an $s$-wave Anderson impurity sitting at three different positions: on top of $\mathrm{C}$, on a bridge site, and in the middle of a hexagon.

At each adsorption site, the spherically symmetric $s$-wave impurity can be modeled by equal hopping matrix element $V$ to its nearest-neighbor sites. For such an Anderson impurity on top of a carbon atom or at a bridge site, we obtain

$$
V_{k}=(V, 0)^{T}, \quad V_{k}=(V, V)^{T},
$$

respectively, by translating that nearest-neighbor hopping into the matrix formalism of Eq. (3) and performing the Fourier transformation. Combining this with Eq. (7) results in $B \sim O\left(E_{\mathrm{F}}\right)$ for $E_{\mathrm{F}} \rightarrow 0$. This situation corresponds to Eq. (11) with $q$ being enhanced as $E_{\mathrm{F}} \rightarrow 0$.

For the impurity in middle of the hexagon the Fouriertransformed hopping reads

$$
V_{k}=\left(\xi^{*}(k), \xi(k)\right)^{T} .
$$

As the dispersion does, this coupling vanishes linearly when approaching the Brillouin-zone corners $K$ and $K^{\prime}$. As a consequence, any possible Kondo resonance due to such an im-

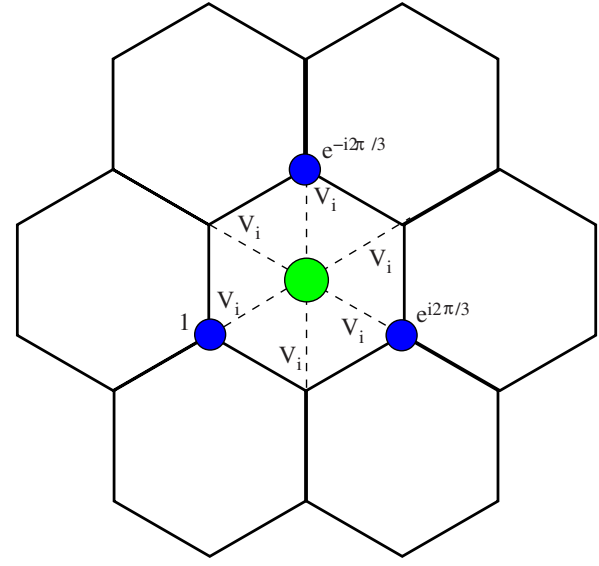

FIG. 2. (Color online) Model of an Anderson impurity in the middle of the graphene hexagon. Electrons from neighboring can hop onto the impurity by the hopping matrix elements $V$. For an electron from the vicinity of the Brillouin-zone corner $K$ phase differences of its wave function at neighboring sites belonging to sublattice A (atoms marked by small blue circles) are given. The sum of these phase factors vanishes.

purity will lead to $q \gg 1$ : Eq. (7) results in $B \sim O\left(E_{\mathrm{F}}^{2}\right)$ for $E_{\mathrm{F}} \rightarrow 0$ in this case and leads to an even stronger enhancement of the $q$ factor than for the impurity on top of carbon or at a bridge site.

The origin of this effect can be understood in terms of the $C_{6 v}$ symmetry as discussed above or in terms of destructive quantum interference in graphene lattice: for each state in the vicinity of $K\left(K^{\prime}\right)$ the phase of the wave function at nearestneighbor sites to impurity winds the phase either clockwise or counterclockwise depending on sublattice, as illustrated in Fig. 2. The phase of the wave function of atoms in sublattice A winds leading to $\xi(k) \rightarrow 0$ for $k \rightarrow K$ or $k \rightarrow K^{\prime}$. This cancellation is a direct result of the Berry phase associated with the Dirac points in graphene: the vanishing of $\xi(k)$ is due to topological properties of the honeycomb lattice as was discussed in Ref. 33.

\section{CONCLUSIONS}

We have studied the Fano asymmetry factors arising in scanning tunneling spectroscopy of impurity resonances in graphene. We find a tendency of $q$ factors being generally enhanced as compared to usual metal surfaces. For the realistic $d$-electron case of Co we performed ab initio calculations addressing the Fano line shapes of Kondo resonances in this system. The crystal field of graphene splits the Co $d$ orbitals into two doublets $E_{1,2}$ and one singlet $A_{1}$ of states. Our calculations suggest that the $E_{1}$ doublet states are responsible for the Kondo effect and for unusual Fano $q$ factors seen in the experiments. ${ }^{19} \mathrm{~A}$ strong dependence of Fano factors on the involved orbitals is found. For an $s$-wave impurity placed in the middle of a hexagon we have shown that the same destructive interference that leads to the linearly vanishing DOS is responsible for an anomalously enhanced $q$ factor. We thus conclude that nontrivial properties of the Kondo effect in graphene is an inherent property of Dirac materials with nodal spectrum. Quantum interference effects, 
like the Fano effect, are extremely sensitive to atomistic details such as symmetries of the involved orbitals or specific impurity positions. Upon completion of this work we became aware of recent works of Zhuang et al., ${ }^{34}$ Uchoa et al., ${ }^{35}$ and Saha et $a l .{ }^{36}$ that address related questions for the case of $s$-wave magnetic impurities.

\section{ACKNOWLEDGMENTS}

We are grateful to D. Arovas, A. Rosch, and J. von Delft for useful discussions. This work was supported by Stichting voor Fundamenteel Onderzoek der Materie (FOM), The Netherlands and by SFB-668(A3), Germany. Work at Los Alamos and Stanford University was supported through BES and LDRD funds under the auspices of NNSA and Office of Basic Energy Sciences of the U.S. Department of Energy under Contracts No. DE-AC52-06NA25396 and No. DEAC02-76SF00515. Computation time at HLRN is acknowledged.
${ }^{1}$ A. C. Hewson, The Kondo Problem to Heavy Fermions (Cambridge University Press, Cambridge, England, 1993).

${ }^{2}$ J. Li, W.-D. Schneider, R. Berndt, and B. Delley, Phys. Rev. Lett. 80, 2893 (1998).

${ }^{3}$ V. Madhavan, W. Chen, T. Jamneala, M. F. Crommie, and N. S. Wingreen, Science 280, 567 (1998).

${ }^{4}$ H. C. Manoharan, C. P. Lutz, and D. M. Eigler, Nature (London) 403, 512 (2000).

${ }^{5}$ N. Knorr, M. A. Schneider, L. Diekhöner, P. Wahl, and K. Kern, Phys. Rev. Lett. 88, 096804 (2002).

${ }^{6}$ L. Limot and R. Berndt, Appl. Surf. Sci. 237, 572 (2004).

${ }^{7}$ N. Néel, J. Kröger, R. Berndt, T. O. Wehling, A. I. Lichtenstein, and M. I. Katsnelson, Phys. Rev. Lett. 101, 266803 (2008).

${ }^{8}$ K. S. Novoselov, A. K. Geim, S. V. Morozov, D. Jiang, Y. Zhang, S. V. Dubonos, I. V. Grigorieva, and A. A. Firsov, Science 306, 666 (2004).

${ }^{9}$ K. S. Novoselov, A. K. Geim, S. V. Morozov, D. Jiang, M. I. Katsnelson, I. V. Grigorieva, S. V. Dubonos, and A. A. Firsov, Nature (London) 438, 197 (2005).

${ }^{10}$ Y. Zhang, Y.-W. Tan, H. L. Stormer, and P. Kim, Nature (London) 438, 201 (2005).

${ }^{11}$ C. R. Cassanello and E. Fradkin, Phys. Rev. B 53, 15079 (1996).

${ }^{12}$ K. Ingersent, Phys. Rev. B 54, 11936 (1996).

${ }^{13}$ M. Hentschel and F. Guinea, Phys. Rev. B 76, 115407 (2007).

${ }^{14}$ K. Sengupta and G. Baskaran, Phys. Rev. B 77, 045417 (2008).

${ }^{15}$ P. S. Cornaglia, G. Usaj, and C. A. Balseiro, Phys. Rev. Lett. 102, 046801 (2009).

${ }^{16}$ A. V. Balatsky, I. Vekhter, and J.-X. Zhu, Rev. Mod. Phys. 78, 373 (2006).

${ }^{17}$ F. Schedin, A. K. Geim, S. V. Morozov, E. W. Hill, P. Blake, M. I. Katsnelson, and K. S. Novoselov, Nature Mater. 6, 652 (2007).

${ }^{18}$ T. O. Wehling, K. S. Novoselov, S. V. Morozov, E. E. Vdovin, M. I. Katsnelson, A. K. Geim, and A. I. Lichtenstein, Nano Lett. 8, 173 (2008).
${ }^{19}$ L. S. Mattos, C. R. Moon, M. W. Sprinkle, C. Berger, K. Sengupta, A. V. Balatsky, W. A. de Heer, and H. C. Manoharan (unpublished).

${ }^{20}$ T. O. Wehling, A. V. Balatsky, M. I. Katsnelson, A. I. Lichtenstein, K. Scharnberg, and R. Wiesendanger, Phys. Rev. B 75, 125425 (2007).

${ }^{21}$ P. R. Wallace, Phys. Rev. 71, 622 (1947).

${ }^{22}$ G. W. Semenoff, Phys. Rev. Lett. 53, 2449 (1984).

${ }^{23}$ V. Madhavan, W. Chen, T. Jamneala, M. F. Crommie, and N. S. Wingreen, Phys. Rev. B 64, 165412 (2001).

${ }^{24}$ M. Plihal and J. W. Gadzuk, Phys. Rev. B 63, 085404 (2001).

${ }^{25}$ Assuming linear dispersion in the entire Brillouin zone and approximating the Brillouin zone by circles around the two nonequivalent Dirac points results in $D \approx 6 \mathrm{eV}$ (Ref. 20).

${ }^{26}$ J. P. Perdew, J. A. Chevary, S. H. Vosko, K. A. Jackson, M. R Pederson, D. J. Singh, and C. Fiolhais, Phys. Rev. B 46, 6671 (1992).

${ }^{27}$ G. Kresse and J. Hafner, J. Phys.: Condens. Matter 6, 8245 (1994).

${ }^{28}$ P. E. Blöchl, Phys. Rev. B 50, 17953 (1994).

${ }^{29}$ G. Kresse and D. Joubert, Phys. Rev. B 59, 1758 (1999).

${ }^{30}$ Y. Yagi, T. M. Briere, M. H. F. Sluiter, V. Kumar, A. A. Farajian, and Y. Kawazoe, Phys. Rev. B 69, 075414 (2004).

${ }^{31}$ Y. Mao, J. Yuan, and J. Zhong, J. Phys.: Condens. Matter 20, 115209 (2008)

${ }^{32}$ B. Amadon, F. Lechermann, A. Georges, F. Jollet, T. O. Wehling, and A. I. Lichtenstein, Phys. Rev. B 77, 205112 (2008).

${ }^{33}$ J. L. Mañes, F. Guinea, and M. A. H. Vozmediano, Phys. Rev. B 75, 155424 (2007).

${ }^{34}$ H.-B. Zhuang, Q.-f. Sun, and X. C. Xie, Europhys. Lett. 86, 58004 (2009).

${ }^{35}$ B. Uchoa, L. Yang, S.-W. Tsai, N. M. R. Peres, and A. H. Castro Neto, Phys. Rev. Lett. 103, 206804 (2009).

${ }^{36}$ K. Saha, I. Paul, and K. Sengupta, arXiv:0906.2788 (unpublished). 